Sharif University of Technology
Scientia Iranica
SCIENTIA
IRAN ICAA

\title{
Effects of stenosis and RBC motion on mass transfer in the microvessels using immersed boundary-lattice Boltzmann method
}

\author{
M. Alafzadeh ${ }^{a}$, E. Shirani ${ }^{b, *}$, E. Yahaghic ${ }^{\text {, and N. Fatouraee }}{ }^{\text {d }}$ \\ a. Department of Mechanical Engineering, Isfahan University of Technology, Isfahan, P.O. Box 8415683111, Iran. \\ b. Foolad Institute of Technology, Fooladshahr, Isfahan, P.O. Box 8491663763, Iran. \\ c. Department of Physics, Imam Khomeini International University, Ghazvin, P.O. Box 3414896818, Iran. \\ d. Department of Biomedical Engineering, Amirkabir University of Technology, Tehran, P.O. Box 1591634311, Iran.
}

Received 14 February 2016; received in revised form 14 January 2017; accepted 17 October 2017

\section{KEYWORDS}

Stenosis microvessel; Lattice Boltzmann method;

Immersed boundary;

Wall shear stress;

RBC deformation;

Permeability.

\begin{abstract}
In this work, for better understanding of microvessels disorders, mass transfer at a stenotic and the straight capillary wall in the presence of RBC motion are investigated. The immersed boundary-lattice Boltzmann method is used for this purpose. The erythrocyte is considered as an immersed biconcave shaped tissue around the capillary as a porous medium. The gamma function for input concentration, which is close to the actual stenosis brain capillary, is used. The simulated results obtained for both stenosis and straight capillaries are compared. It is shown that while the RBC motion has negligibly small effects on wall mass transfer in straight capillaries, its effect is not negligible at stenosis capillaries.

(C) 2018 Sharif University of Technology. All rights reserved.
\end{abstract}

\section{Introduction}

Microvessels stenosis is an abnormal narrowing of a blood microvessel, which may cause heart attack and brain stroke. It is important to understand the detailed hemodynamic characteristics of blood flow passing through a stenosed microvessel and its effects on mass transfer, influencing the pathogenesis and development of circulatory disease. It should be mentioned that changes in microvessel morphology are typical of conditions for predisposition to stroke [1]. In fact, investigation of microcirculation in various pathological conditions from a biomechanical point of view would assist in disease diagnosis and devising therapeutic techniques [2]. Due to the importance of blood flow in a

\footnotetext{
*. Corresponding author. Tel.: +98 31526366ry
} E-mail address: eshirani@ictp.it (E. Shirani)

doi: $10.24200 /$ sci. 2017.4549 stenosed microvessel, various studies have been carried out. Wang and Xing [2] investigated the traverse of erythrocyte through a 2-D microchannel with stenosis. They modeled the erythrocyte as membrane particles connected by springs. Their simulation considered erythrocyte with various shapes and stiffnesses to mimic the healthy and abnormal cells. Dimakopoulos et al. [3] studied non-Newtonian blood flow in stenotic rigid vessel. They used finite element method to present the mathematical modeling. Vahidkhah et al. [4] showed the flow of deformable red blood cells in stenotic microvessels. They demonstrated the effects of Fahraeus and Fahraeus-Lindqvist in stenosed microvessels. Jiang et al. [5] performed numerical investigation of hemodynamics of RBCs in microvessels with stenosis. They used the numerical model based on the immersed boundary-fictitious domain method. Xiao et al. [6] simulated the behavior of a single RBC flowing through a microvessel stenosis by dissipative particle dynamics method. They studied the mechan- 
ical behavior of the $\mathrm{RBC}$ in the microvessel stenosis. $\mathrm{Xu}$ et al. [7] simulated the behavior of a haemocyte when passing through stenotic capillary by immersed boundary. They analyzed the RBC deformation and obtained the velocity and pressure profiles in the capillary.

Magnetic Resonance Imaging (MRI) is a medical imaging technique used to investigate the anatomy and physiology of bodies in both health and disease. It can be used to diagnose the microvascular disease in the body. Usually, a radioactive substance or contrast agent is used as an indicator in MRI [8]. There are also many efforts based on the capability of MRI to evaluate brain tumors and other abnormalities in the body [8]. Johnson and Wilson [9] introduced the Tissue Homogeneity ( $\mathrm{TH})$ model for the capillary. $\mathrm{TH}$ model is one of two models for simulation of vascular permeability. In this model, tissue is divided into Extra-Vascular Surface (EVS) and Intra-Vascular Surface (IVS). These spaces are separated by a vessel wall whose Permeability Surface (PS) area product is used as a measure of vascular permeability to a given tracer. Yahaghi et al. [8] presented a new method based on Monte Carlo to estimate the leakage of a contrast agent out of a capillary. It should be noted that all the above-mentioned efforts were made for studying the behavior of RBCs flowing in stenosed microvessel, or estimating the leakage of a contrast agent out of a microvessel. However, most of these reports did not consider the effects of RBCs flowing through microvessel stenosis on mass permeability. To our knowledge, up to now, there is very little work on the effects of stenosis on mass transfer in microvessel.

The objective of this work is to demonstrate the effects of stenosis on RBCs motion, Wall Shear Stress (WSS), and consequently, the contrast agent permeability in the brain capillary. The lattice Boltzmann method is used to numerically simulate the flow in this study. Because of its remarkable capability in simulation of complex flows, such as deformable suspended particle in the fluid, the lattice Boltzmann method is implemented for simulation of RBC motion and the contrast agent transfer in the capillary stenosis.

In this work, first, a brief overview of immersed boundary-lattice Boltzmann method (LBM) is presented. Next, to validate our method and results, the diffusion of contrast agent around a straight capillary is simulated and the results are compared with the previously obtained numerical results. Finally, the flow of plasma with suspended RBC and the contrast agent transfer are simulated in 2-D brain capillary stenosis. Investigation of the flow with $3-\mathrm{D}$ models is limited by their computationally expensive nature. As such, various researchers have employed 2-D models to efficiently and qualitatively study microvessels flows [10]. Furthermore, the presented results are compared with the previous 2-D statistical results.

It should be mentioned that the medium around the capillary is considered as a porous medium. Moreover, the effects of stenosis and RBC deformation on mass transfer are presented.

\section{Computational domain}

In the present study, the dynamic process of 2-D RBC flows and the transport of the contrast agent (GdDTPA) through a straight microvessel and a stenosed one (with a diameter of $H=9 \mu \mathrm{m}$ ) are considered as illustrated in Figure 1. It should be mentioned that the symmetrical stenosis is located in $x=0.37 \mathrm{~L}$ with stenosis severity of $\frac{R}{H / 2} * 100=30 \%$ and $50 \%$ ( $R$ is the radius of stenosis) as shown in Figure 1(b).

Plasma is considered as Newtonian, laminar, and incompressible flow. In microvessel, the flow velocity for transporting the material between plasma and cells is low [11] and the Reynolds number is less than or equal to 0.001 . The density and viscosity of the blood plasma are $\rho=1025 \mathrm{~kg} / \mathrm{m}^{3}$ and $v=$ $1.17 \times 10^{-6} \mathrm{~m}^{2} / \mathrm{s}$, respectively. A fully developed velocity profile at the inlet of the capillary lumen and constant pressure at the outlet of capillary are assumed. At the interface between the lumen and the tissue around the capillary, constant filtration velocity is applied. Other boundary conditions are shown in Figure 1. The physical parameters are used as shown in Table 1.

For the mass transfer, the input function is modeled by the following gamma function [8]:

$$
c_{i n}=\frac{A}{\Gamma(k)} t^{k} e^{-t}
$$

where $t$ is time, and $k$ and $A$ are constants, which are the position and amplitude of the peak of the gamma function (12 and 2.5), respectively.

At the interface between the lumen and the tissue around the capillary, the concentration boundary condition for mass diffusion across the wall is [12]:

Table 1. Physical parameters for Gd-DTPA transfer in brain capillary [11].

\begin{tabular}{ccccccc}
\hline $\begin{array}{c}\boldsymbol{\rho}_{\text {plasma }} \\
\left(\mathbf{k g} / \mathbf{m}^{\mathbf{3}}\right)\end{array}$ & $\begin{array}{c}\boldsymbol{D}_{\text {contrast }} \\
\left(\mathbf{m}^{\mathbf{2}} / \mathbf{s}\right)\end{array}$ & $\begin{array}{c}\boldsymbol{\nu}_{\text {plasma }} \\
\left(\mathbf{m}^{\mathbf{2}} / \mathbf{s}\right)\end{array}$ & $\begin{array}{c}\text { Diameter } \mathrm{RBC} \\
(\boldsymbol{\mu} \mathbf{m})\end{array}$ & $\begin{array}{c}\text { Diameter }_{\text {capillary }} \\
(\boldsymbol{\mu} \mathbf{m})\end{array}$ & $\mathbf{S c}$ & $\mathbf{R e}$ \\
\hline 1025 & $0.4 \times 10^{-9}$ & $1.17 \times 10^{-6}$ & 8 & 9 & 2926.8 & 0.001 \\
\hline
\end{tabular}




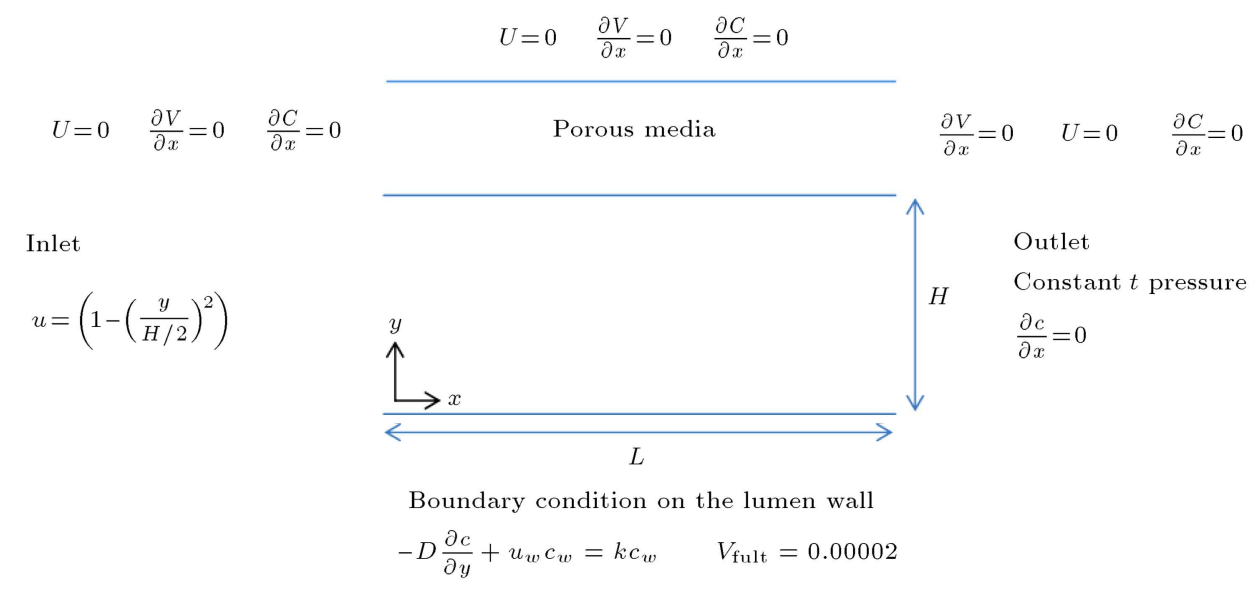

(a)

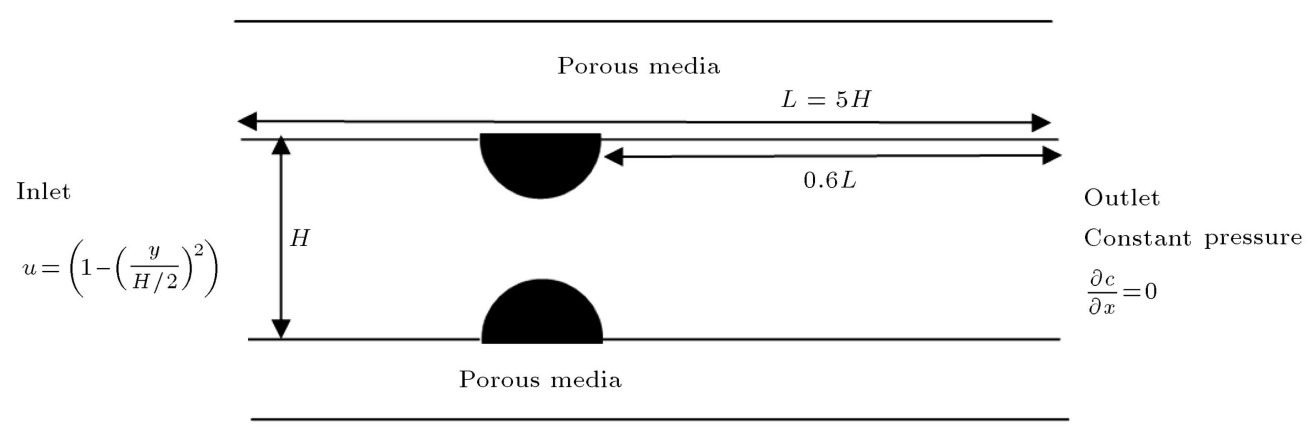

(b)

Figure 1. Schematics of the capillary: (a) The straight capillary, and (b) the stenotic capillary.

$$
-D \frac{\partial c}{\partial y}+u_{w} c_{w}=k c_{w}
$$

where $D$ is the diffusion coefficient, $k$ is the permeability at the wall, and $c_{w}$ and $u_{w}$ are the solute concentration and velocity on the wall, respectively.

In addition, zero normal diffusive mass flux is employed at the lumen outlet, and inlet and outlet of the capillary tissue. The boundary conditions for the flow in stenosed capillary are the same as those for the capillary without stenosis.

\section{Numerical method}

In this article, the LBM with Multi-Relaxation-Time (MRT) scheme proposed by d'Humi $\mu$ eres [13] is chosen to simulate the flow of plasma in two-dimensional capillaries. The MRT model has more flexibility than the Single Relaxation Time (SRT) in choosing the relaxation times. When viscosity increases, the relaxation time increases and the lattice Boltzmann method is not accurate. By using the MRT scheme, the accuracy increases. It should be mentioned that there is no need to use MRT for mass transfer, because the relaxation time, i.e., the Schmidt number, for mass transfer is not high and using the single relaxation technique, SRT, to simulate mass transfer is sufficient.
It should be mentioned that the Schmidt number, Sc, is chosen to be more than 2000 in this article, thus the viscosity is much higher than the diffusion coefficient.

For simulation of the flow in extra-vascular space (the tissue around the capillary) as the porous medium, the MRT scheme proposed by Liu et al. [14] is employed. For 2-D geometry, D2Q9 model is used as a lattice to for descretizing the fluid field.

The SRT-LBM proposed by Takaji et al. [15] is used for modeling of the mass transfer. The lattice Boltzmann method should be modified for mass transfer in porous media. The related equations can be derived as [16]:

$$
\begin{aligned}
& g_{\alpha}\left(x+e_{\alpha} \delta t, t+\delta t\right)-g_{\alpha}(x, t)=\frac{g_{\alpha}^{(\mathrm{eq})}(x, t)-g_{\alpha}(x, t)}{\tau_{c}}, \\
& g_{\alpha}{ }^{\mathrm{eq}}=\omega_{\alpha} C\left[\varepsilon+\frac{e u}{c_{s}^{2}}\right] \\
& \varepsilon C=\sum_{\alpha} g_{\alpha}
\end{aligned}
$$

where $g_{\alpha}(x, t)$ is the concentration distribution function, $g_{\alpha}^{\text {eq }}(x, t)$ is the corresponding equilibrium state, $\tau_{c}$ is the relaxation time for mass transfer, $\varepsilon$ is porosity, $C$ is concentration, $\delta t$ is time steps, $c_{s}$ is the speed of 
sound, $\omega_{\alpha}$ is weighting coefficient, and $e_{\alpha}$ is particle velocity vector defined as [16]:

$$
\begin{aligned}
& e_{0}=(0,0), \\
& e_{\alpha}=c\left(\cos \frac{(i-1) \pi}{2}, \sin \frac{(i-1) \pi}{2}\right) \\
& \alpha=1,2,3,4 \\
& e_{\alpha}=c \sqrt{2}\left(\cos \left(\frac{(i-5) \pi}{2}+\frac{\pi}{4}\right), \sin \left(\frac{(i-5) \pi}{2}+\frac{\pi}{4}\right)\right), \\
& \alpha=5,6,7,8
\end{aligned}
$$

In porous media, the diffusion coefficient is defined as [16]:

$$
D=2 \varepsilon c_{s}^{2}\left(\tau_{c}-\frac{1}{2}\right) \delta t .
$$

In this work, an RBC is modeled as a 2-D biconcave capsule as follows [17]:

$$
\begin{aligned}
\bar{y}= & 0.5\left(1-\bar{x}^{2}\right)^{0.5}\left(c_{0}+c_{1} \bar{x}^{2}+c_{2} \bar{x}^{4}\right) \\
& -1<\bar{x}^{2}<1
\end{aligned}
$$

in which $c_{0}=0.207, c_{1}=2.002$, and $c_{2}=1.122$, and the non-dimensional coordinates, $\bar{x}, \bar{y}$, are $x / 3.91$ and $y / 3.91$, respectively.

In the present study, the Immersed Boundary Method (IBM) is used to simulate the RBC deformations. The forces acting on the membrane consist of three parts: elastic force, bending force, and interaction force.

To understand the effects of bending and elastic forces on the behavior of $\mathrm{RBC}$, the following two dimensionless groups are defined as:

$$
\begin{aligned}
& E_{B}=\frac{E_{b}}{E_{s} a^{2}}, \\
& G=\frac{\mu U_{m}}{E_{s}},
\end{aligned}
$$

where $a$ is the radius of the RBC, $U_{m}$ is the mean velocity of plasma, $E_{s}$ is the membrane elastic modulus, and $E_{b}$ is the membrane bending resistance. $G$ shows the competition between the viscous force in the plasma flow and the elastic resistance force of the membrane. $E_{B}$ is the ratio of the membrane elasticity to its bending resistance.

To show the interaction force, the Morse potential is used as [18]:

$$
\begin{aligned}
& \varphi(r)=D_{e}\left[e^{2 \beta\left(r_{0}-r\right)}-2 e^{\beta\left(r_{0}-r\right)}\right], \\
& f(r)=-\frac{\partial \varphi(r)}{\partial r}=2 D_{e} \beta\left[e^{2 \beta\left(r_{0}-r\right)}-e^{\beta\left(r_{0}-r\right)}\right] .
\end{aligned}
$$

In this approach, $\varphi(r)$ is interactional energy and $f(r)$ is the interaction force between two nodes on the membrane. $r$ is the surface separation, $r_{0}$ is the zero force separation, $D_{e}$ is the surface energy, and $\beta$ is a scaling factor [18]. To study the effect of Morse potential force on mass transfer, another dimensionless group can be defined as:

$$
D_{E}=\frac{D_{e}}{E_{s}} .
$$

\section{Boundary conditions}

In this article, constant velocity and pressure, which are implemented by Zou-He method [19], are used at straight boundaries. To implement the constant velocity on curved boundaries (stenosis), the method proposed by Guo et al. [20] is used.

For mass transfer on straight boundary, the method proposed by Wang et al. [21] is chosen and for curved boundary, Guo et al. [20] method is employed.

The hydrodynamic force induced by a fluid flowing over a wall surface consists of the pressure and the shear stress. The shear stress for incompressible flow is $[22]$ :

$$
\begin{aligned}
\tau_{i j}= & \left(1-\frac{1}{2 \tau}\right) \sum_{\alpha} f_{\alpha}^{(\text {neq })}(x, t) \\
& \left(e_{\alpha, i} e_{\alpha, j}-\frac{1}{D} e_{\alpha} \cdot e_{\alpha} \delta_{i j}\right),
\end{aligned}
$$

where $f_{\alpha}$ is distribution function, $D$ shows the dimension, and $f^{\text {neq }}=f-f^{\text {eq }}$.

\section{Results and discussion}

To validate the presented results, first, Gd-DTPA transfer in the abnormal brain's straight capillary $(k=$ $0.27 \mathrm{ml} / \mathrm{min} / \mathrm{g}$ is the permeability of the brain wall) is considered (see Figure 1(a)). The concentration in the middle of tissue (as the function of time) as well as the input concentration is shown in Figure 2. It should be mentioned that the average concentration along the vertical axis is used in this figure. The contrast agent diffuses into the tissue around the brain capillary due to some brain diseases. As it is shown in Figure 2(a), the used function is Arterial Input Function (AIF) for the brain capillary, which looks fit to the real data. The concentration of the GdDTPA in the EVS increases from zero to maximum and then decreases by the fresh blood that enters the brain capillary. The contrast agent concentrations in the EVS are in good agreement with the Monte Carlo results and the actual concentration extracted from dynamic contrast enhancement images [8]. The present results in Figure 2 show that LBM can be used to 


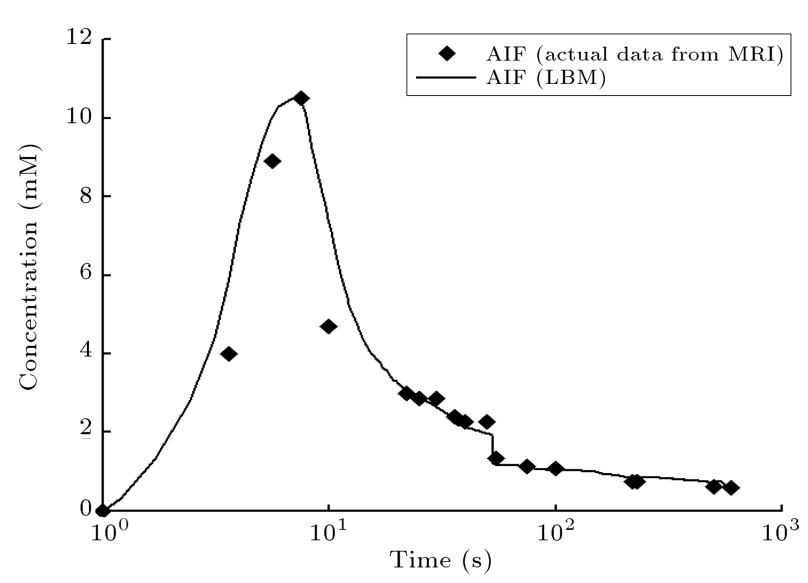

(a)

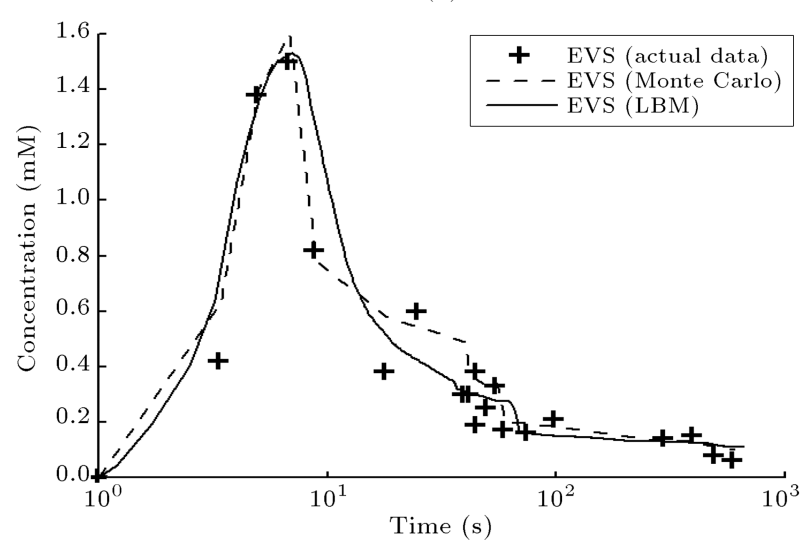

(b)

Figure 2. Contrast agent concentration (a) as the arterial input function, and (b) in the EVS for the abnormal brain capillary in comparison with the actual data extracted from MRI and Monte Carlo results ( $K_{\text {Darcy }}=$ $\left.6.6 \times 10^{-13} \mathrm{~m}^{2}, \varepsilon=0.4\right)$.

accurately simulate the contrast agent transfer in the brain capillary and the tissue around it.

The results of grid study are shown in Figure 3, where the ratio of outlet to inlet concentration is given for different non-dimensional width sizes. This normalized concentration is computed for the abnormal capillary with $k=0.5 \mathrm{ml} / \mathrm{min} / \mathrm{g}$. As shown, when the mesh width is less than 0.2 , the results are independent of $H / L$.

As pointed out previously, the simulation of mass transfer in the brain capillary with stenosis is the main aim of this paper.

To investigate the sensitivity of our results to stenosis severity in the simulation capillary, the variations of normalized wall shear stress magnitude in the axial direction of the stenosis are shown in Figure 4 for two different stenosis severities (30\% and 50\%). A normalized WSS is defined as [23]:

$$
\tau_{w}^{*}=\frac{\tau_{w}}{P_{x} H / 2},
$$

where $\tau_{w}$ is wall shear stress, $H$ is the height of channel,

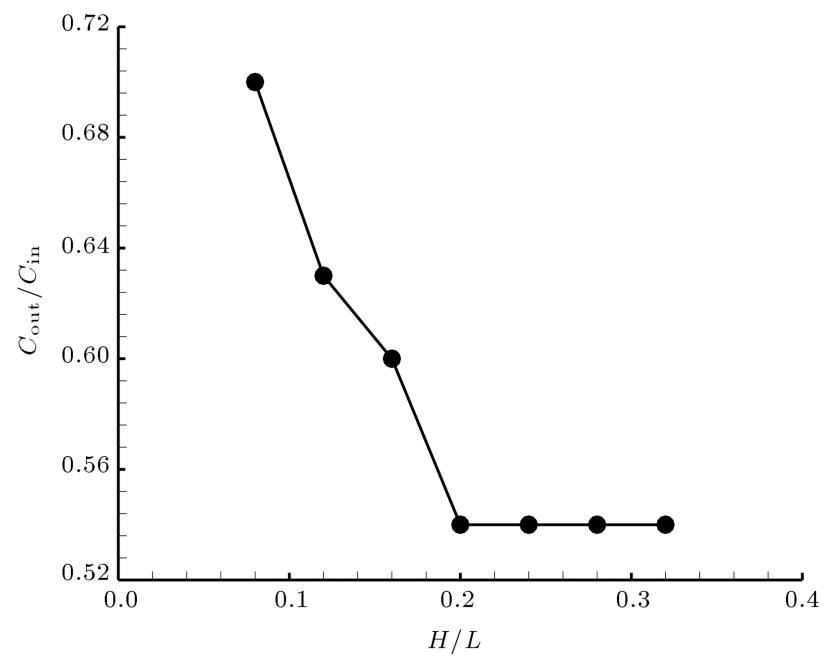

Figure 3. Normalized concentration as the function of mesh width.

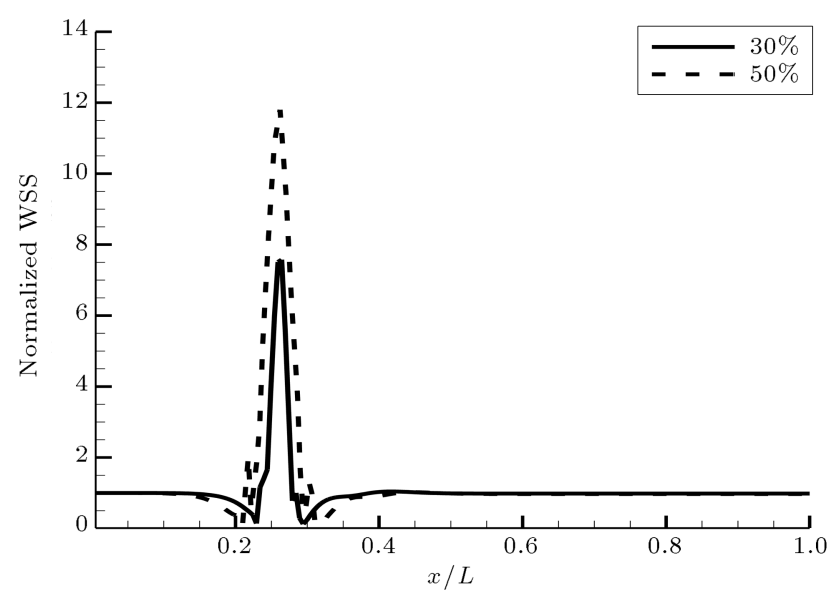

Figure 4. WSS for stenosis severities of $30 \%$ and $50 \%$ along the capillary wall.

and $P_{x}$ is the pressure gradient along the horizontal axis.

WSS increases in the stenosis due to the velocity increase at this point and, then, the reduction in the velocity passing the stenosis peak reduces WSS along the axial axis. As it is presented in Figure 4, variation of WSS magnitude is symmetric along the stenosis due to the symmetrical streamline. It should be mentioned that the Reynolds number is very small in the capillary $(\operatorname{Re}=0.001)$. For small $R e$, when inertia is not important, the flow is smooth and laminar, so there are not any vortices behind the stenosis and the streamline is symmetric. In this situation, there is no separation point as shown in Figure 4 . When stenosis severity increases, variation of velocity is higher and WSS increases (see Figure 4), so the value of WSS for $50 \%$ severity is larger than its value for $30 \%$ severity. Figure 5 shows the normalized concentration along the stenosis for two stenosis severities. The concentration decreases along the luminal surface due to the contrast 


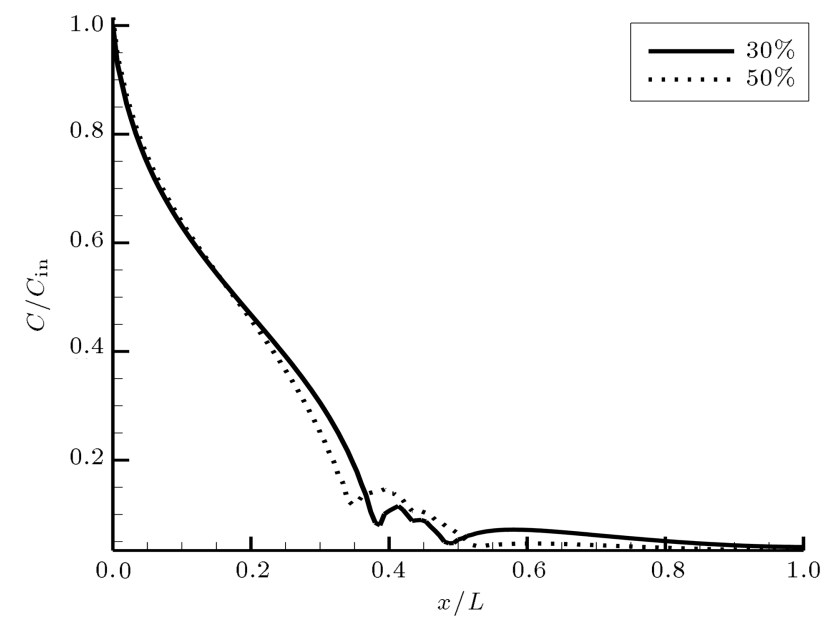

Figure 5. Normalized concentration along the capillary wall for stenosis severities of $30 \%$ and $50 \%$.

agent permeability on the wall. But at the throat of the stenosis, the Gd-DTPA concentration on the capillary wall increases due to the accelerated flow and, consequently, stronger convection. When severity increases, the convection becomes stronger, and the value of concentration is larger for $50 \%$ severity than for $30 \%$ severity. The results show that Gd-DTPA flux across the endothelium is reduced in the low WSS region. Variations of WSS have strong effect on mass transfer across the endothelium in the microvessels such as capillaries.

Also, because of the variation of WSS along the stenosis, diffusion of Gd-DTPA in the tissue around the brain capillary changes. The normalized concentration in the middle of the tissue is plotted in Figure 6 as a function of time for $30 \%$ severity and for straight capillary.

The maximum diffusion of Gd-DTPA in the middle of tissue is a function of severities. It means that severity affects the mass diffusion in the tissue

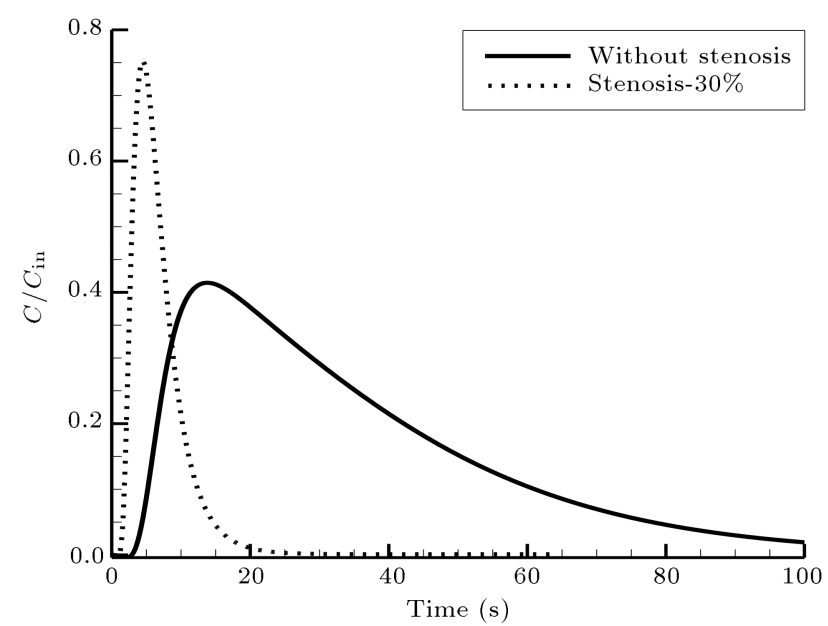

Figure 6. Normalized concentration in the EVS for stenosis severity of $30 \%$. by changing WSS on the capillary wall. Since higher severity increases the wall shear stress and mass concentration on the wall, it is clear that it increases the maximum amount of Gd-DTPA in the tissue (as the function of WSS). In addition, the normalized concentration reaches the maximum value faster when there is stenosis in the capillary. This result refers to the increase in mass diffusion due to the existence of stenosis in the capillary. In other words, stenosis causes higher convection and mass flux increases in this region. Therefore, more Gd-DTPA diffuses to the tissue around the capillary in less time.

One of the effective parameters on the wall shear stress is RBCs motion in the vessels. The existence of an RBC in the channel increases the system's hydrodynamic resistance [23]. To further investigate the variation of mass transfer associated with the variation of WSS caused by RBC motion, the RBC is placed in the center of capillary.

The first case is a single red blood cell vertically traversing the straight capillary without stenosis $(k=$ $0.5 \mathrm{ml} / \mathrm{min} / \mathrm{g}$ ). RBC motion can lead to the maximum value of shear stress due to a larger velocity gradient across the cell-wall gap at the front of an RBC. Then, WSS relaxes down and reaches a maximum point at the end of the cell in the wake flow region behind the cell. Another peak for WSS value is observed when the shear stress recovers to its normal value [23]. The effect of WSS variation due to the RBC motion on mass transfer is depicted in Figure 7. The results show that although the RBC motion changes the wall shear stress along the capillary, the effect of WSS on Gd-DTPA transfer is less than that of permeability effect. Therefore, RBC motion does not have any effect on mass transfer in the abnormal capillary without stenosis.

The next case considered is that the RBC is located in the capillary with stenosis for $k=$

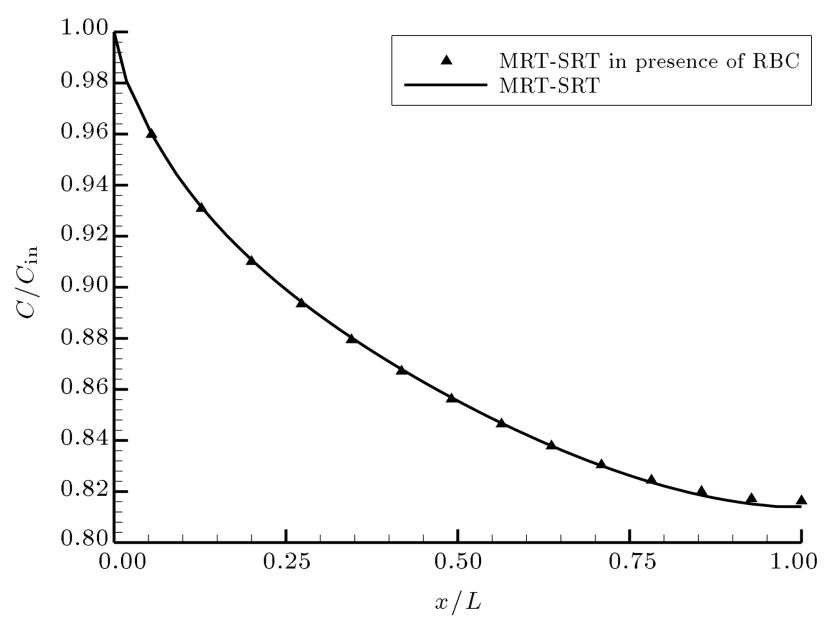

Figure 7. Normalized concentration along the capillary wall with/without the presence of RBC for $k=0.5$ $\left(G=0.076, E_{B}=1.89 \times 10^{-5}\right)$. 


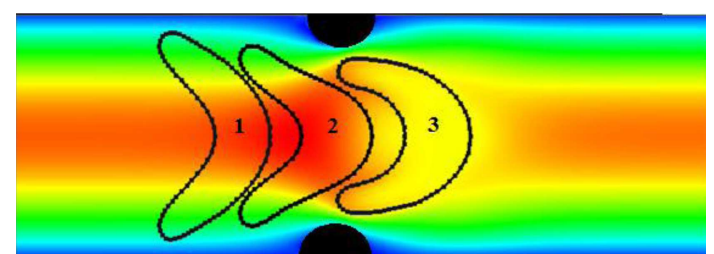

Figure 8. Deformation of single RBC flowing traverses through stenosis $\left(G=0.076, E_{B}=1.89 \times 10^{-5}\right)$.

$0.5 \mathrm{ml} / \mathrm{min} / \mathrm{g}$. First, the RBC movements and deformations in the stenotic capillary are analyzed. Figure 8 shows the RBC behavior in the stenotic capillary with $30 \%$ severity.

This Figure shows the snapshots of RBC traversing three locations in the brain capillary: 1) at the front of stenosis, 2) at the stenosis throat, and 3) at the back of stenosis. The results show that the cell is deformed under the hydrodynamic forces. At the stenosis throat, it further stretches into a parachute shape due to the squeezing and the RBC recovers its biconcave shape upon exiting the stenosis. To study the effect of RBC behavior passing stenosis on WSS, the next case considered is the investigation of the effect of RBC motion on the distribution of WSS along the capillary.

Figure 9 shows the WSS variations at the capillary wall for $30 \%$ severity for different locations of RBC. When RBC is at different regions, WSS increases at the same region.

Although RBC motion in different regions has effect on WSS at the same region, the results show that RBC motion generally increases the wall shear stress. As pointed out previously, RBC motion causes two peaks and a valley in WSS profile in the straight capillary [23] and the first peak appears in front of the $\mathrm{RBC}$, which is shown in Figure 9 for $\mathrm{RBC}$ in different locations.

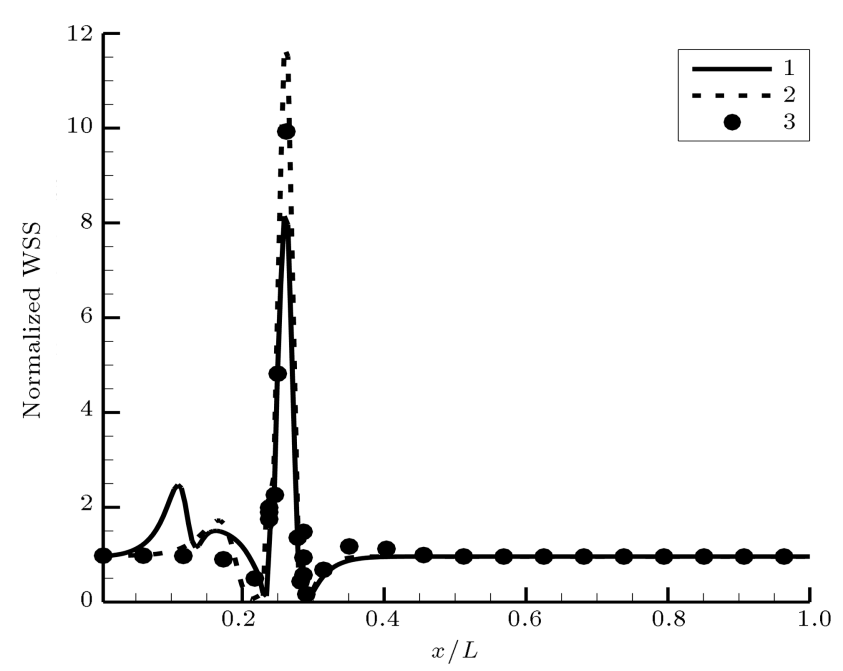

Figure 9. Variation of WSS along the stenosis as the function of RBC location.
Moreover, the variations of Gd-DTPA concentration along the stenosis with the existence of RBC (in locations 1, 2, and 3) are presented in Figure 10. According to this figure, the mass transfer in the stenotic capillary is closely related to the RBC behav-

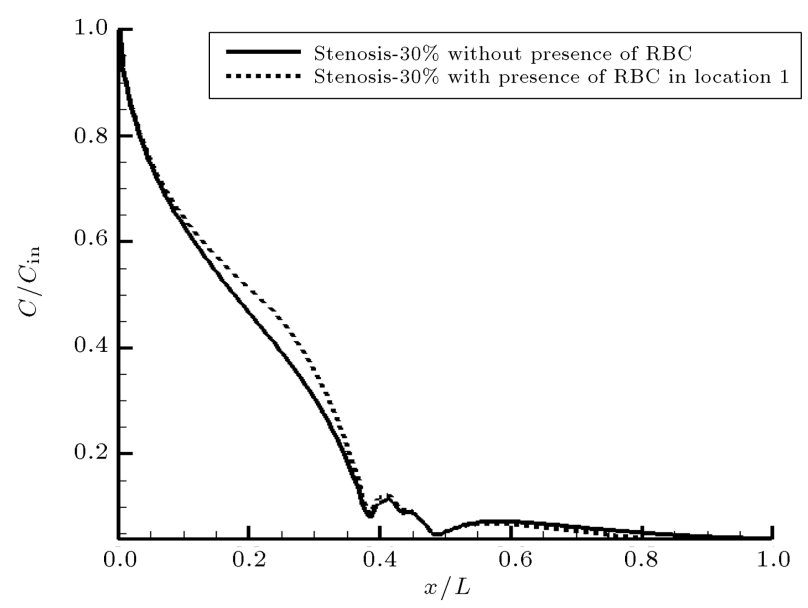

(a)

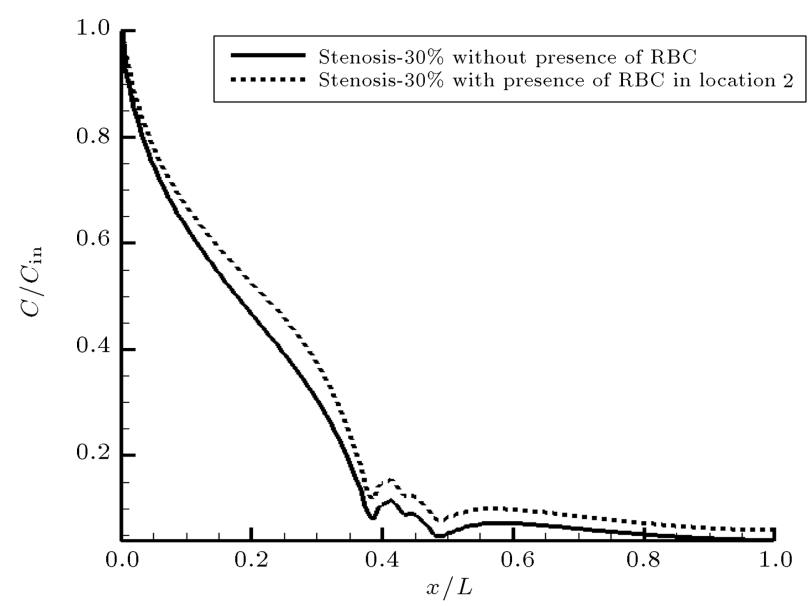

(b)

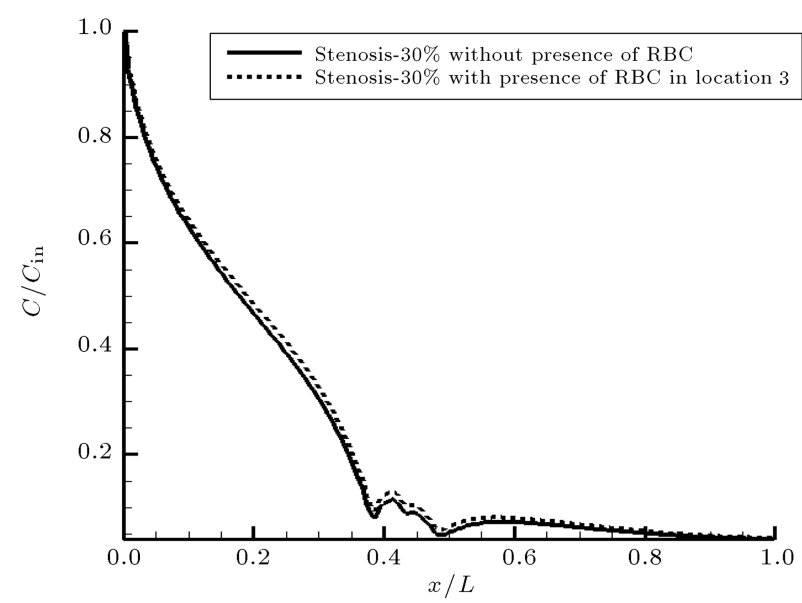

(c)

Figure 10. Normalized concentration along the stenosed capillary wall as the function of the RBC motion at (a) location 1 , (b) location 2, and c) location 3. 
ior. The cell passing the stenosis changes the WSS distribution (see Figure 9) and WSS increases the GdDTPA concentration on the wall. In other words, WSS variations caused by $\mathrm{RBC}$ motion impose higher mass flux across the capillary wall.

Thus, it is shown that RBC deformation passing locations 1 and 2 in the stenotic capillary is more likely to affect the mass transfer on the wall than the RBC passing location 3 is. As mentioned in Figure 8, the RBC stretches into the parachute shape when moving through the stenosis. Therefore, the distance between the RBC and luminal concentration on the wall becomes longer when it leaves stenosis and the effect of RBC motion on luminal surface concentration is lower.

The results show the difference between the contrast agent transfer in the straight and stenotic capillaries. The stenosis increases the velocity and WSS, so the effect of WSS cannot be neglected in the place of stenosis. Therefore, the parameters which affect the WSS can also change the mass transfer along the capillary wall.

\section{Conclusion}

The lattice Boltzmann method was utilized to study the effect of stenosis and RBC motion passing stenosis on Gd-DTPA transfer in the capillary. The motion and deformation of RBC in the stenotic capillary were studied by coupling the immersed boundary and LBM. In this paper, variation of Gd-DTPA (the contrast agent that is used in MRI) concentration was explored as the function of WSS variation. It was also depicted that the stenosis and RBC motion were the parameters which had effects on the distribution of WSS along the stenotic capillary and consequently, they changed mass transfer on the capillary wall. The results showed that although they changed the wall shear stress, $\mathrm{RBC}$ motion and deformation did not have any effect on mass transfer in the straight capillary. In fact, in the straight capillary, the velocity was less than diffusion velocity and permeability, so the effect of WSS could be neglected. It should be noticed that in the stenotic capillary, WSS is more than that in the straight capillary. So, its effects on mass transfer cannot be neglected. Finally, the results showed that the LBM had good potential applications in biology for some disease diagnoses.

\section{Nomenclature}

$\begin{array}{ll}h & \text { Capillary diameter } \\ L & \text { Capillary length } \\ \delta & \text { Thickness of EVS } \\ \rho & \text { Density }\end{array}$

$\begin{array}{ll}\nu & \text { Viscosity } \\ \operatorname{Re} & \text { Reynolds number } \\ K & \text { Permeability } \\ C_{w} & \text { Solute concentration on the capillary } \\ & \text { lumen wall } \\ u_{w} & \text { Velocity on the wall } \\ D_{e} & \text { Effective diffusion coefficient } \\ C_{s} & \text { Speed of sound } \\ K_{\text {Darcy }} & \text { Permeability of tissue } \\ \varepsilon & \text { Porosity of tissue } \\ g_{\alpha} & \text { Concentration distribution function } \\ g_{\alpha}^{\text {eq }} & \text { Equilibrium concentration distribution } \\ & \text { function } \\ \tau_{c} & \text { Relaxation time for mass transfer } \\ \tau & \text { Relaxation time } \\ \omega_{\alpha} & \text { Weighting coefficient } \\ \delta t & \text { Time steps } \\ e_{\alpha} & \text { Particle velocity vector }\end{array}$

\section{References}

1. Stergaard, L., Jespersen, S.N., Mouridsen, K., et al. "The role of the cerebral capillaries in acute ischemic stroke: the extended penumbra model", Journal of Cerebral Blood Flow \& Metabolism, 33, pp. 635-648 (2013).

2. Wang, T. and Xing, Z.W. "Erythrocyte hemodynamics in stenotic microvessels: A numerical investigation", Physical Review E, 88, pp. 1-9 (2013).

3. Dimakopoulos, Y., Kelesidis, G., Tsouka, S., Georgiou, G.C., and Tsamopoulos, J. "Hemodynamics in stenotic vessels of small diameter under steady state conditions: effect of viscoelasticity and migration of red blood cells", Biorheology, 52, pp. 183-210 (2015).

4. Vahidkhah, K., Balogh, P., and Bagchi, P. "Flow of red blood cells in stenosed microvessels", Scientific Rep., 6, pp. 1-15 (2016).

5. Jiang, X.M., Tong, W., and Zhongwen X. "Simulation study of hemodynamics of red blood cells in stenotic microvessels", Advanced Materials Research, 647, pp. 321-324 (2013).

6. Xiao, L.L., Chen, S., Lin, C.S., and Liu, Y. "Simulation of a single red blood cell flowing through a microvessel stenosis using dissipative particle dynamics", Mol Cell Biomech., 11(1), pp. 67-85 (2014)

7. Yuan-Qing, X., Xiao-Ying, T., Fang-Bao, T., Yu-Hua, P., Yong, X., and Yan-Jun, Z. "IB-LBM simulation of the haemocyte dynamics in a stenotic capillary", Computer Methods in Biomechanics and Biomedical Engineering, 17(9), pp. 978-985 (2014).

8. Yahaghi, E., et al., "Estimation of contrast agent concentration in intra-and extra-vascular spaces of brain tissue", Mathematical Biosciences, 204, pp. 102118 (2006). 
9. Johnson, J.A. and Wilson, T.A. "A model for capillary exchange", Am. J. Physiol., 210, pp. 1299-1303 (1966).

10. Ye, S.S., Ng, Y.C., Tan, J., Leo, H.L., and Kim, S. "Two-dimensional strain-hardening membrane model for large deformation behavior of multiple red blood cells in high shear conditions", Theoretical Biology and Medical Modelling, 11, pp. 11-19 (2014).

11. Guyton Arthur, C. and Hall, J.E., Textbook of Medical Physiology, Elsevier Saunders (2006).

12. Yang, N. and Vafai, K. "Modeling of low-density lipoprotein (LDL) transport in the artery-effects of hypertension", International Journal of Heat and Mass Transfer, 49, pp. 850-867 (2006).

13. D'Humi $\mu$ eres D., "Generalized lattice Boltzmann equations in rarefied gas dynamics: theory and simulations", Prog. Aeronaut. Astronaut, 159, pp. 450-458 (1992).

14. Liu, Q., He, Y.L., Li, Q., and Tao, W.Q. "A multiplerelaxation-time lattice Boltzmann Model for convection heat transfer in porous media", International Journal of Heat and Mass Transfer, 73, pp. 761-775.

15. Inammuro, T., Yoshino, M., Inoue, H., Mizuno, R., and Ogino F. "A lattice Boltzmann method for a binary miscible fluid mixture and its application to a heat-transfer problem", Journal of Computational Physics, 179, pp. 201-215 (2002).

16. Vafai, K., Porous Media Application in Biological Systems and Biotechnology, Taylor $\mathscr{E}$ Francis Group (2011).

17. Evans, E.A. and Fung, Y.C. "Improved measurements of the erythrocyte geometry", Microvasc. Res., 4, pp. 335-347 (1972).

18. Vahidkhah, K. and Fatouraee, N. "Numerical simulation of red blood cell Behavior in a stenosed arteriole using the immersed boundary- Lattice Boltzmann method", Int. J. Numer. Meth. Biomed. Engng., 28, pp. 239-256 (2011).

19. He, X. and Zou, Q. "Analysis and boundary condition of the lattice Boltzmann BGK model with two velocity components", Journal of Statistical Physics, 87, pp. 115-136 (1995).
20. Guo, Zh., Zhang, Ch., and Shi, B. "An extrapolation method for boundary conditions in lattice Boltzmann method", Physics of Fluids, 14(6), pp. 2007-2010 (2002).

21. Wang, J., Wang, M., and Li, Zh. "A lattice Boltzmann algorithm for fluid-solid conjugate heat Transfer", International Journal of Thermal Sciences, 46, pp. 228-234 (2007).

22. Filippova, O. and Hanel, D. "Grid refinement for lattice- BGK models", J. Comput. Phys., 147, pp. 219228 (1998).

23. Xiong, W. and Zhang, J. "Shear stress variation induced by red blood cell Motion in microvessel", Annals of Biomedical Engineering, 38(8), pp. 26492659 (2010).

\section{Biographies}

Mina Alafzadeh received her $\mathrm{PhD}$ degree in Mechanical Engineering from Isfahan University of Technology in 2016. Her research interests include biomechanics.

Ebrahim Shirani received his $\mathrm{PhD}$ degree from Stanford University in 1981. He was a Faculty Member in Mechanical Engineering Department at Isfahan University of Technology, Isfahan, Iran, from 1981 to 2011. He is now Faculty Member and Vice President of Foolad Institute of Technology, Fooladshahr, Isfahan, Iran. He is also Editor in Chief of Journal of Applied Fluid Mechanics and Fellow of Iranian Academy of Science, and has published more than 300 journal and conference papers.

Effat Yahaghi received her PhD degree from AmirKabir University of Technology in 2006. She is Associate Professor of Physics at Imam Khomeini International University, Ghazvin.

Nasser Fatouraee received his $\mathrm{PhD}$ degree from Laval University in 2000. He is Associate Professor of Biomechanics at Amirkabir University of Technology (Polytechnic Tehran) and president of Iranian society for Biomedical Engineering. 